\title{
Numerical Modeling of Plasma Manipulation Using an ExB Layer in a Hypersonic Boundary Layer
}

\author{
Minkwan Kim * and Iain D. Boyd ${ }^{\dagger}$ \\ University of Michigan, Ann Arbor, MI, 48109 \\ Michael Keidar $\ddagger$ \\ George Washington University, Washington, D.C, 20052
}

When a vehicle flies with hypersonic velocity or re-enters the atmosphere, a weakly ionized plasma layer is generated around the vehicle due to the shock heated air. Since the created plasma layer has a high plasma number density, the vehicle has a communication problem known as radio blackout. In this study, we illustrate that an applied ExB layer can manipulate the plasma density in a specific region. The manipulated plasma reduces radio wave attenuation in the plasma layer and provides the possibility for communication during radio blackout. The possibility of the ExB layer mitigation scheme is evaluated in a realistic operating condition for a hypersonic flow in terms of signal attenuation.

\section{Nomenclature}

$\begin{array}{ll}\overrightarrow{\mathbf{B}} & \text { Magnetic field } \\ C_{s} & \text { Species mass fraction } \\ c_{s} & \text { Electrical chage of the species } \\ D_{s} & \text { Species diffusion coefficient } \\ \overrightarrow{\mathbf{E}} & \text { Electric field } \\ E_{t} & \text { Total energy } \\ \mathbf{F} & \text { Flux vector } \\ \mathbf{F}_{v} & \text { Viscous flux vector } \\ f & \text { Frequency, [Hz] } \\ h_{s} & \text { Species enthalpy } \\ \overrightarrow{\mathbf{J}}_{s} & \text { Species diffusion flux } \\ \overrightarrow{\mathbf{j}} & \text { Current density } \\ k_{r} & \text { Chemical reaction coefficient } \\ m_{e} & \text { Electron mass, } 9.109 \times 10^{-31} \mathrm{~kg} \\ m_{s} & \text { Species molecular mass } \\ n & \left.\text { Number density, [m }{ }^{-3}\right] \\ p & \text { Pressure } \\ \mathbf{Q} & \text { Vector of conserved variables } \\ \overrightarrow{\mathbf{q}} & \text { Heat flux } \\ R_{r} & \text { Chemical reaction rate } \\ \mathbf{S}_{c v} & \text { Thermochemical source term } \\ \mathbf{S}_{M H D} & \text { Electromagnetic source term } \\ u & \text { x-direction flow velocity } \\ v & \text { y-direction flow velocity }\end{array}$

\footnotetext{
*Graduate Student, Department of Aerospace Engineering, minkwan@umich.edu, AIAA Student Member.

$\dagger$ Professor, Department of Aerospace Engineering, iainboyd@umich.edu, AIAA Associate Fellow.

$¥$ Assistant Professor of Engineering and Applied Science, Department of Mechanical and Aerospace Engineering, keidar@gwu.edu, AIAA Associate Fellow.
} 


$\begin{array}{ll}w & \text { Z-direction flow velocity } \\ \alpha & \text { Ion-slip coefficient } \\ \beta & \text { Hall parameter } \\ \lambda & \text { Bulk viscosity coefficient } \\ \vec{\lambda} & \text { Magnetic field stream function } \\ \mu & \text { Viscosity coefficient } \\ \rho & \text { Mixture mass density } \\ \rho_{i} & \text { Species density } \\ \sigma & \text { Electrical conductivity } \\ \tilde{\sigma} & \text { Conductivity tensor } \\ \tau_{i, j} & \text { Viscous stress } \\ \phi & \text { Potential } \\ \omega & \text { Frequency, [rad/s] } \\ \dot{\omega}_{s} & \text { Species mass production rates of chemical reactions } \\ \text { Subscripts } \\ b & \text { Backward reaction } \\ f & \text { Forward reaction } \\ i & \text { Species } \\ p & \text { Plasma } \\ r a d i o & \text { Radio wave }\end{array}$

\section{Introduction}

A PLASMA layer, formed around a vehicle during a hypersonic or reentry flight, usually has a high electron Anumber density, $10^{15}$ to $10^{19} \mathrm{~m}^{-3}$ depending on altitude and velocity of vehicles. ${ }^{1}$ The created plasma layer reflects or attenuates radio waves which are transmitted from or to a vehicle because of its high plasma number density. ${ }^{2}$ This makes a temporary communication interruption, which is known as radio blackout. As a result of radio blackout, a vehicle loses voice communication, GPS navigation, mission command, and electric countermeasure capability. For reentry vehicles, radio blackout typically lasts several minutes, depending on the angle of reentry and the particular trajectory. ${ }^{3}$ For example, the Soyuz TMA reentry vehicle experiences about 10 minutes of radio blackout and the Mars Pathfinder experienced a 30 second radio blackout during its Mars entry mission. Hypersonic cruise vehicles operating within the atmosphere would also experience intermittent or continuous blackout depending on altitude and velocity. ${ }^{4}$

Developing solutions that allow communication through a plasma layer are a high priority because of flight safety, catastrophe analysis, and mission success. ${ }^{5}$ During 10 minutes of radio blackout, a hypersonic vehicle could fly thousands of miles without guidance from a ground station or GPS satellite. It is clearly a flight safety issue for a manned hypersonic or reentry vehicle. Radio blackout also makes catastrophe analysis impossible, eliminating a critical factor for understanding and preventing reentry accidents. In the space shuttle Columbia disaster, telemetry was lost prior to disintegration due to radio blackout. There was little available data at the ground station to assist in finding the cause of the disaster. For an unmanned vehicle, a blackout makes the vehicle lose the control or guidance of a ground station. In this case, continuous and real-time telemetry determines whether the vehicle succeeds in a mission or not. Therefore, continuous communication and real-time telemetry is very important for both manned and unmanned vehicles, for flight safety, catastrophe analysis and mission success.

There are three dominant mission scenarios which require communication through a plasma layer. These are manned or unmanned re-entry vehicles, air-breathing hypersonic cruise vehicles, and ballistic missiles. ${ }^{4}$ The same general physics can be used to describe the blackout problem in all three scenarios. Radio blackout occurs when the plasma frequency of the plasma layer around a vehicle exceeds the radio wave frequency used for communications: $f_{p}>f_{\text {radio }}$. The plasma frequency $f_{p}$ is calculated from: ${ }^{6}$

$$
f_{p}=\frac{\omega_{p}}{2 \pi} \approx 8.9683 \times n_{e}^{\frac{1}{2}}
$$

where $n_{e}$ is the plasma density, in units of particles per $\mathrm{m}^{3}$. To lower the plasma frequency, it is necessary to reduce the plasma number density.

During the last 50 years, a number of approaches have been suggested for mitigation of hypersonic radio 
blackout, including aerodynamic shaping, quenchant injection, high radio frequencies, high transmission power, and Raman scattering. One of the most promising methods is the magnetic window method ${ }^{7}$ where a strong DC magnetic field is used to allow radio wave propagation through the plasma layer. This technique is limited, however, by the weight of magnets required for such transmission. We expand upon the magnetic window via the addition of electric fields to increase the density reduction possible for a given magnetic field strength. The reduced plasma density can create a "window" for communication. A radio wave can propagate through a plasma layer without disturbing the flow field of a vehicle in the hypersonic or reentry flight condition via a "window" created by an applied ExB field. ${ }^{6}$

Figure 1 shows a schematic of an applied ExB layer in a hypersonic plasma layer. The applied electric field accelerates the plasma temporarily over the antenna, reducing the local plasma density to allow communication. The plasma returns to its original velocity and density past the mitigation system. However, the use of an electric field alone is impractical due to shielding of the electrodes by sheath effects. At high densities, the sheath which forms around the electrodes can have a thickness of millimeters or less. The electrode shielding problem can be improved by a magnetic field. The presence of a magnetic field can trap the electrons, allowing the existence of a strong electric field. This produces ion acceleration through regions of the hypersonic plasma layer, resulting in a corresponding plasma density decrease. The reduced plasma density creates a "window" in the hypersonic plasma layer through which radio waves can be transmitted.

The plasma density distribution will be affected by the magnitudes and profiles of the magnetic and electric fields. ${ }^{3,6}$ There are still limits on the density of plasma which can be affected, placed by practical limitations on the magnetic and electric fields which can be applied because of magnet weight and electrode arcing. ${ }^{6}$ However, a significant range of densities can be mitigated, corresponding to increased altitude range of communications or decreased periods of radio blackout. Therefore our numerical analysis can help to estimate the plasma density distribution and establish the optimal ExB layer configuration to allow communication. In this study, we develop a numerical model for simulation of plasma manipulation by using an ExB layer in the hypersonic flow.

In previous studies, we suggested one-dimensional and two-dimensional ExB layer models for a single species plasma without a chemistry model. ${ }^{3,6}$ The suggested model demonstrated that an applied ExB layer can significantly reduce the plasma density under an experimental condition using an argon plasma. However, the single species plasma model cannot be easily applied to simulate the re-entry hypersonic flight condition, thus requiring the consideration of a multi-chemical species model. This study focuses mainly on the simulation of the ExB layer in a hypersonic flow, to assess the mitigation scheme in a realistic operating condition. In Sec. II, we describe a numerical model of an ExB layer in hypersonic flow. The geometry of the OREX hypersonic vehicle with an ExB layer and boundary conditions are described in Sec. III. Section IV shows numerical results of the OREX geometry with an ExB layer. The results are evaluated in terms of blackout mitigation in Sec. V. Conclusions are formulated in Sec. VI.

\section{Numerical Modeling}

This study simulates hypersonic flow with an ExB layer as a radio blackout mitigation scheme. Figure 2 shows the thermochemical properties of the OREX re-entry flight. As can be seen, the re-entry flow requires a multi-species chemistry model. ${ }^{8}$ In this study, the following eleven species air chemistry model is considered: $\mathrm{N}, \mathrm{O}, \mathrm{N}_{2}, \mathrm{O}_{2}, \mathrm{NO}, \mathrm{N}^{+}, \mathrm{O}^{+}, \mathrm{N}_{2}^{+}, \mathrm{O}_{2}^{+}, \mathrm{NO}^{+}$, and $e^{-}$.

The governing equations for the $\mathrm{ExB}$ layer with the eleven chemical species are composed mainly of two parts, which are the flow field and the electric field. The flow field equations describe a hypersonic flow with a Lorentz force, which is provided by the applied ExB layer. In this case, the flow field equations require the current, electric field, and magnetic field. These are provided by the electric field equation which comes from Maxwell's equations and Faraday's law.

\section{A. Flow Field Equations}

The flow field equations describe a hypersonic flow with the ExB layer and they are similar to the NavierStokes equations and are solved numerically using a hypersonic CFD code, LeMANS (The Michigan Aerothermal Navier-Stokes Solver), developed at the University of Michigan Nonequilibrium Gas and Plasma Dynamics Laboratory (NGPDL). The hypersonic condition causes many physical phenomena in the flow field including chemical non-equilibrium, vibrational and electronic excitation, thermal non-equilibrium and ion- 
ization. ${ }^{9}$ This study considers chemical non-equilibrium using a finite rate chemistry model and assumes a thermal equilibrium consistent with the thermochemical properties of the OREX re-entry as shown in Figure $2 .{ }^{10}$ Therefore, the hypersonic flow is described by the continuum framework by means of a chemical non-equilibrium one-temperature gas model.

The governing equations for the flow field description are the mass conservation equations of the chemical species, the momentum conservation equations and the total energy conservation equation. In order to simplify the flow field equations, the magnetic Reynolds number is assumed to be much smaller than unity because the typical magnetic Reynolds number is the order of $10^{-3}$ in a weakly ionized gas. ${ }^{11}$ For a low magnetic Reynolds number assumption, the flow field equations are obtained by coupling the Navier-Stokes equation with the Lorentz force, $\vec{J} \times \vec{B}$, and the energy interaction, $\vec{E} \cdot \vec{J}$, terms. ${ }^{12}$

The governing equations of the flow field with the ExB layer can be expressed in the following form:

$$
\frac{\partial \mathbf{Q}}{\partial t}+\nabla \cdot \mathbf{F}-\nabla \cdot \mathbf{F}_{v}=\mathbf{S}_{c v}+\mathbf{S}_{M H D}
$$

where $\mathbf{Q}$ is the vector of conserved variables, $\mathbf{F}$ is the flux vector, $\mathbf{F}_{v}$ is the viscous flux vector, $\mathbf{S}_{c v}$ is a thermochemical source term, and $\mathbf{S}_{M H D}$ is a source term related to the electromagnetic force. For a three-dimensional system, the vector of conserved variables, $\mathbf{Q}$, is given by

$$
\mathbf{Q}=\left\{\begin{array}{c}
\rho_{1} \\
\rho_{2} \\
\vdots \\
\rho_{n s} \\
\rho u \\
\rho v \\
\rho w \\
E_{t}
\end{array}\right\}
$$

where $\rho_{i}$ is the density of species $i, u, v$, and $w$ are flow velocities, and $E_{t}$ is the total energy.

The inviscid and diffusive flux vector components in the $\mathrm{x}$ direction are given by

$$
\mathbf{F}_{x}=\left\{\begin{array}{c}
\rho_{1} u \\
\rho_{2} u \\
\vdots \\
\rho_{n s} u \\
\rho u^{2}+p \\
\rho u v \\
\rho u w \\
(E+p) u
\end{array}\right\}
$$

and

$$
\mathbf{F}_{v, x}=\left\{\begin{array}{c}
-J_{x, 1} \\
-J_{x, 2} \\
\vdots \\
-J_{x, n s} \\
\tau_{x x} \\
\tau_{x y} \\
\tau_{x z} \\
\tau_{x x} u+\tau_{x y} v+\tau_{x z} w-q_{x}-\sum_{\text {all species }}\left(J_{x, s} h_{s}\right)
\end{array}\right\}
$$

where, $\rho$ is the mixture mass density which is defined as the summation of species mass densities, $\rho=$ $\sum_{\text {all species }} \rho_{s}, p$ is the pressure, $\overrightarrow{\mathbf{J}}_{s}$ is the species diffusion flux, $\tau_{i, j}$ are the viscous stress components, $\overrightarrow{\mathbf{q}}$ is the heat flux, and $h_{s}$ is the species enthalpy. The diffusion fluxes are modeled using a modified Ficks law to enforce that the sum of the diffusion fluxes is zero ${ }^{9,13}$ 


$$
\overrightarrow{\mathbf{J}}_{s, \text { modified }}=\overrightarrow{\mathbf{J}}_{s}-C_{s} \sum_{r \neq e} \overrightarrow{\mathbf{J}}_{s}
$$

with

$$
\overrightarrow{\mathbf{J}}_{s}=-\rho D_{s} \nabla C_{s}
$$

where $D_{s}$ is the species diffusion coefficient and $C_{s}$ is the species mass fraction. However, for the electrons, Eq. 6 gives a small value for the electron diffusion because the mass of electrons is relatively small compared to that of atoms and molecules as shown in Table 1. In this case, the diffusion flux of electrons can be calculated assuming ambipolar diffusion which guarantees the charge neutrality of the flow field by ${ }^{9}$

$$
\overrightarrow{\mathbf{J}}_{e}=m_{e} \sum_{s \neq e} \frac{\overrightarrow{\mathbf{J}}_{s, \text { modified }} c_{s}}{m_{s}}
$$

where $m_{e}$ is the electron mass, $9.109 \times 10^{-31} \mathrm{~kg}, c_{s}$ is the electrical charge of the species, and $m_{s}$ is the species molecular mass. The viscous stress tensor, $\tilde{\tau}$ can be expressed for a Newtonian viscous fluid as follows: ${ }^{14}$

$$
\tau_{i, j}=\mu\left(\frac{\partial u_{i}}{\partial x_{j}}+\frac{\partial u_{j}}{\partial x_{i}}\right)+\delta_{i, j} \lambda \nabla \overrightarrow{\mathbf{V}}
$$

where $\mu$ is the coefficient of viscosity and $\lambda$ is the coefficient of bulk viscosity. The bulk viscosity coefficient can be obtained using Stokes' hypothesis. ${ }^{9,14}$

$$
\lambda+\frac{2}{3} \mu=0
$$

The detailed expressions for the diffusion and viscosity coefficients can be found in Ref. 9 .

The thermochemical source term, $\mathbf{S}_{v c}$, are given by

$$
\mathbf{S}_{c v}=\left\{\begin{array}{c}
\dot{\omega}_{1} \\
\dot{\omega}_{2} \\
\vdots \\
\omega_{n s} \\
0 \\
0 \\
0 \\
0
\end{array}\right\}
$$

where $\dot{\omega}_{s}$ is the species mass production rates of chemical reactions, and can be expressed as ${ }^{8}$

$$
\dot{\omega}_{s}=m_{s} \sum_{r}^{\text {all reaction }}\left(\beta_{s, r}-\alpha_{s, r}\right)\left(R_{f, r}-R_{b, r}\right)
$$

where $\alpha$ and $\beta$ are the stoichiometric coefficients of the reactions, and $R_{f, r}$ and $R_{b, r}$ are the forward and backward reaction rates, respectively. The forward reaction rate, $R_{f, r}$, is defined by

$$
R_{f, r}=k_{f, r} \prod_{s}^{\text {all species }}\left(\frac{\rho_{s}}{m_{s}}\right)^{\alpha_{s, r}}
$$

where $k_{f, r}$ is the forward reaction coefficient. The backward reaction rate, $R_{b, r}$, is defined by

$$
R_{b, r}=k_{b, r} \prod_{s}^{\text {all species }}\left(\frac{\rho_{s}}{m_{s}}\right)^{\beta_{s, r}}
$$

where $k_{b, r}$ is the backward reaction coefficient. The details of the forward and backward reaction coefficients can be found in Ref. 9 and 15. 
The source term for the electromagnetic force can be expressed in the following form,

$$
\mathbf{S}_{M H D}=\left\{\begin{array}{c}
0 \\
0 \\
\vdots \\
0 \\
(\overrightarrow{\mathbf{j}} \times \overrightarrow{\mathbf{B}})_{x} \\
(\overrightarrow{\mathbf{j}} \times \overrightarrow{\mathbf{B}})_{y} \\
(\overrightarrow{\mathbf{j}} \times \overrightarrow{\mathbf{B}})_{z} \\
\overrightarrow{\mathbf{E}} \cdot \overrightarrow{\mathbf{j}}
\end{array}\right\}
$$

where $\overrightarrow{\mathbf{j}}$ is the current density vector, $\overrightarrow{\mathbf{B}}$ is the magnetic field vector, and $\overrightarrow{\mathbf{E}}$ is the electric field vector.

The flow field equations, Eq. 2, are solved by LeMANS, which is a chemically reacting, parallelized, implicit Navier-Stokes CFD code using a modified Steger-Warming Flux Vector Splitting scheme to calculate inviscid fluxes between mesh volumes. ${ }^{9}$ To include the electromagnetic effect, additional source terms of the electromagnetic effect, $\mathbf{S}_{M H D}$, are included in LeMANS.

\section{B. Electric Field Equations}

The equations that describe the flow field have three electrical quantities, $\overrightarrow{\mathbf{j}}$, $\overrightarrow{\mathbf{B}}$, and $\overrightarrow{\mathbf{E}}$. To obtain these quantities, it is necessary to use an electric field equation.

First, the magnetic field $\overrightarrow{\mathbf{B}}$, is obtained from the imposed magnetic field. In the electric field equation, the magnetic Reynolds number, $R_{M}$, is a good parameter for characterizing the equation because it is a measure of the coupling between the motion of the magnetic field line and the motion of the conducting fluid. ${ }^{16}$ When the magnetic Reynolds number is much smaller than unity, the magnetic field is not greatly affected by the fluid motion. This means that the magnetic field induced by current is negligible compared with the imposed magnetic field. In this study, the magnetic Reynolds number of the hypersonic flow field is assumed very small. This assumption is quite reasonable because in a Newtonian limit, the magnetic Reynolds number becomes very small in hypersonic flow, usually on the order of $10^{-3}$ for many aerospace applications. ${ }^{17-19}$ The magnetic Reynolds number is smaller than $10^{-3}$ when the ionization rate is less than $1 \%{ }^{11}$ In the OREX case at $79.9 \mathrm{~km}$ altitude condition, the maximum ionization rate is $0.07 \%$. Therefore, the magnetic field, $\overrightarrow{\mathbf{B}}$, is a known variable from the imposed magnetic field. The configuration of the imposed magnetic field can be calculated using a divergence free magnetic field condition ${ }^{20}$ with measured magnetic field data as boundary conditions:

$$
\nabla \cdot \overrightarrow{\mathbf{B}}=0
$$

This constraint expresses the absence of magnetic monopoles. In order to calculate the magnetic field from Eq. 16, a magnetic field stream function, $\lambda$, is employed which is calculated from

$$
\overrightarrow{\mathbf{B}}=\nabla \times \vec{\lambda}
$$

where $\vec{\lambda}=(0, \lambda, 0)$. The magnetic stream function is constant along a magnetic field line. In other words, the magnetic stream function indicates the direction of the magnetic field. The definition of the magnetic stream function gives a Poisson equation from the divergence free condition, Eqn. 16 as follows:

$$
\begin{aligned}
\nabla \cdot \overrightarrow{\mathbf{B}} & =\nabla \cdot(\nabla \times \vec{\lambda}) \\
& =0
\end{aligned}
$$

Solving Eq. 18 gives the magnetic-field distribution for the ExB layer.

Equation 18 is solved using the alternating direction implicit (ADI) method. The boundary conditions for Eq. 18 are taken from magnetic-field data measured in the Plasmadynamics and Electric Propulsion Laboratory (PEPL) at the University of Michigan. ${ }^{21,22}$ 
Second, the current density can be obtained from the generalized Ohm's law: ${ }^{23}$

$$
\overrightarrow{\mathbf{j}}=\sigma\left[(\overrightarrow{\mathbf{E}}+\overrightarrow{\mathbf{U}} \times \overrightarrow{\mathbf{B}})-\beta \frac{\overrightarrow{\mathbf{j}} \times \overrightarrow{\mathbf{B}}}{B}+\alpha(\overrightarrow{\mathbf{j}} \times \overrightarrow{\mathbf{B}} \times \overrightarrow{\mathbf{B}})\right]
$$

where $\sigma$ is the electrical conductivity, $\beta$ is the Hall parameter, and $\alpha$ is an ion-slip coefficient.

The generalized Ohm's law, Eq.19 can be simplified with the conductivity tensor as follows:

$$
\overrightarrow{\mathbf{j}}=\tilde{\sigma} \cdot(\overrightarrow{\mathbf{E}}+\overrightarrow{\mathbf{U}} \times \overrightarrow{\mathbf{B}})
$$

where $\tilde{\sigma}$ is a conductivity tensor given by

$$
\tilde{\sigma}=\frac{\sigma}{D}\left[\begin{array}{lll}
\sigma_{11} & \sigma_{12} & \sigma_{13} \\
\sigma_{21} & \sigma_{22} & \sigma_{23} \\
\sigma_{31} & \sigma_{32} & \sigma_{33}
\end{array}\right]
$$

where,

$$
D=\left(1+\alpha \sigma \overrightarrow{\mathbf{B}}^{2}\right)^{2}+(\beta \sigma \overrightarrow{\mathbf{B}})^{2}
$$

and the coefficients, $\sigma_{i j}$, can be expressed as:

$$
\begin{aligned}
\sigma_{11} & =\left(1+\alpha \sigma \overrightarrow{\mathbf{B}}^{2}\right)\left(1+\alpha \sigma B_{x}^{2}\right)+\beta^{2} \sigma^{2} B_{x}^{2} \\
\sigma_{12} & =\sigma\left[\alpha B_{x} B_{y}\left(1+\alpha \sigma \overrightarrow{\mathbf{B}}^{2}\right)-\beta\left(B_{z}-\beta \sigma B_{x} B_{y}\right)\right] \\
\sigma_{13} & =\sigma\left[\alpha B_{x} B_{z}\left(1+\alpha \sigma \overrightarrow{\mathbf{B}}^{2}\right)+\beta\left(B_{y}+\beta \sigma B_{x} B_{z}\right)\right] \\
\sigma_{21} & =\sigma\left[\alpha B_{x} B_{y}\left(1+\alpha \sigma \overrightarrow{\mathbf{B}}^{2}\right)+\beta\left(B_{z}+\beta \sigma B_{x} B_{y}\right)\right] \\
\sigma_{22} & =\left(1+\alpha \sigma \overrightarrow{\mathbf{B}}^{2}\right)\left(1+\alpha \sigma B_{y}^{2}\right)+\beta^{2} \sigma^{2} B_{y}^{2} \\
\sigma_{23} & =\sigma\left[\alpha B_{y} B_{z}\left(1+\alpha \sigma \overrightarrow{\mathbf{B}}^{2}\right)-\beta\left(B_{x}-\beta \sigma B_{y} B_{z}\right)\right] \\
\sigma_{31} & =\sigma\left[\alpha B_{x} B_{z}\left(1+\alpha \sigma \overrightarrow{\mathbf{B}}^{2}\right)-\beta\left(B_{y}-\beta \sigma B_{x} B_{z}\right)\right] \\
\sigma_{32} & =\sigma\left[\alpha B_{y} B_{z}\left(1+\alpha \sigma \overrightarrow{\mathbf{B}}^{2}\right)+\beta\left(B_{x}+\beta \sigma B_{y} B_{z}\right)\right] \\
\sigma_{33} & =\left(1+\alpha \sigma \overrightarrow{\mathbf{B}}^{2}\right)\left(1+\alpha \sigma B_{z}^{2}\right)+\beta^{2} \sigma^{2} B_{z}^{2}
\end{aligned}
$$

The electric field, $\overrightarrow{\mathbf{E}}$, can be obtained from the current density conservation.

$$
\nabla \cdot \overrightarrow{\mathbf{j}}=0
$$

Now, using the generalized Ohm's law:

$$
\nabla \cdot[\tilde{\sigma} \cdot(\overrightarrow{\mathbf{E}}+\overrightarrow{\mathbf{U}} \times \overrightarrow{\mathbf{B}})]=0
$$

Equation 33 becomes a scalar equation by using the definition of electric field, $\overrightarrow{\mathbf{E}}=-\nabla \phi$, as follows:

$$
\nabla \cdot(\tilde{\sigma} \cdot \nabla \phi)=\nabla \cdot(\overrightarrow{\mathbf{U}} \times \overrightarrow{\mathbf{B}})
$$

For a hypersonic re-entry flow, Eq. 34 can be simplified. In Eqs. 19 and 20, the Hall and ion-slip effects are considered. The estimated electron Hall parameter, defined as the product of the electron cyclotron frequency and the mean collision time with the other chemical species, is of the order of $10^{-1} .^{24}$ Therefore, in the plasma region produced behind the shock, the ion slip effect does not have a considerable influence on the electric current. ${ }^{8,25}$ Since the Hall effect does not have a negative influence on the usefulness of MHD 
flow control, the Hall effect should be negligible. ${ }^{25}$ Therefore, the conductivity tensor becomes a scalar and the equation for the electric field is obtained as follows:

$$
\nabla \cdot(\sigma \nabla \phi)=\nabla \cdot[\sigma(\overrightarrow{\mathbf{U}} \times \overrightarrow{\mathbf{B}})]
$$

Equation 35 is solved by using a finite element method (FEM) to give the potential distribution. ${ }^{25,26}$

\section{OREX}

For this study, the OREX (Orbital Re-entry Experiment Vehicle) geometry is considered in order to simulate a hypersonic flow of a re-entry vehicle with an ExB layer to manipulate the plasma because it is a typical blunt body reentry vehicle. OREX, which was launched from the Tanegashima Space Center of Japan in 1994 in order to conduct reentry experiments, used the C-band for the tracking signal, and S-band and X-band for the data telemetry. ${ }^{27}$ OREX was specifically designed to acquire the following data related to atmospheric re-entry:

1. Aerodynamic and aerodynamic heating data during re-entry

2. Heat-resistant structural data during re-entry

3. Communications blackout data during re-entry

4. GPS navigation data during re-entry and in orbit

The simulation of an ExB layer in hypersonic flow of the OREX geometry can show the effectiveness of an ExB layer as a blackout mitigation method in terms of density reduction ratio and signal attenuation.

\section{A. OREX Geometry}

Figure 3 shows the geometry of OREX which has an axisymmetric shape with the locations of electrostatic probes and antenna. OREX is a typical blunt body reentry vehicle with a nearly zero angle of attack in the re-entry experiment. The forebody shape of OREX is composed of a spherical nose with a $1.35 \mathrm{~m}$ radius, a cone, and a circular shoulder.

Figure 4 shows OREX with a proposed configuration of the electrodes and electromagnet that provide the ExB layer for the blackout mitigation scheme. The ExB layer is applied to OREX to manipulate the plasma layer. As can be seen, the electrodes are located on the vehicle surface and they are separated by $10 \mathrm{~cm}$. The electromagnet is located inside of the vehicle. The applied magnetic field data are based on measured data, and is similar to the magnetic field presented in our previous study. ${ }^{21}$

Figure 5 shows the simulation domain with a simplified OREX geometry. The simplified configuration is composed of a spherical nose with a $1.35 \mathrm{~m}$ radius and a cone. Figure 5 also shows the location of the two $5 \mathrm{~mm}$ radius electrodes and the electromagnet. The electromagnet is located at the center of the vehicle because of its size. In order to provide $0.5 \mathrm{~T}$, a typical size of electromagnet is about $60 \mathrm{~cm}$ in diameter. ${ }^{28}$

\section{B. Boundary Conditions}

Table 2 shows the inflow conditions and wall temperature of OREX at several altitudes. ${ }^{29}$ The chemical composition of free stream air is assumed to be $79 \% \mathrm{~N}_{2}$ and $21 \% \mathrm{O}_{2}$ in terms of mole fraction at all altitudes. Since OREX experienced a radio blackout at approximately $80 \mathrm{~km},{ }^{30}$ this study chose the $79.9 \mathrm{~km}$ inflow condition of OREX for the simulation.

The boundary condition of the cathode is straightforward because the potential there is assumed to be an externally given value. The anode is grounded on the vehicle so it has the same potential as the surface. On the surface of OREX, a zero potential is assumed. The inflow and outflow boundary conditions use a zero potential variation across the boundary.

Figure 6 shows the magnetic field configuration. In this study, the electromagnet is assumed to be located at the center of OREX when considering the size of the actual electromagnet which has an approximately $0.5 \mathrm{~m}$ radius for the core. ${ }^{28}$ As can be seen, the maximum magnetic field strength occurs at the front of the vehicle. 


\section{Simulation Results}

Figure 7 shows the electron number density distribution for OREX at $79.9 \mathrm{~km}$. As can be seen, the plasma number density is high enough to cause the radio blackout of L-band and UHF communication. The critical densities for L-band and S-band communications are $3.53 \times 10^{16} \mathrm{~m}^{-3}$ and $6.54 \times 10^{16} \mathrm{~m}^{-3}$, respectively. This result is consistent with the fact that the blackout of OREX actually started at $80 \mathrm{~km}$ and ended at $50 \mathrm{~km} .{ }^{30}$ Figure 8 shows the comparison with the OREX measurement of electron number densities by an electrostatic probe mounted on the conical flank before the probe shoulder ${ }^{29}$ and the simulation result of LeMANS and indicates that LeMANS underestimates the electron number density of the OREX reentry. Since LeMANS provides a three times lower electron number density than the measured data, the plasma density reduction for the real application should be three times stronger than a requirement that is based on the simulation results.

Figure 9 shows the plasma number density distribution with the ExB layer mitigation scheme. Since high electric power is required in order to provide the $0.5 \mathrm{~T}$ magnetic field, the $0.1 \mathrm{~T}$ magnetic field with $-800 \mathrm{~V}$ potential drop is applied in this study in order to illustrate an effectiveness of an ExB layer as a blackout mitigation scheme. As can be seen, the applied ExB layer reduces the electron number density and creates a window for communication. The manipulated plasma number density is low enough for the S-band communication which was used for the data telemetry of OREX. Figure 9 also shows that the plasma density returns to its original density past the applied ExB layer. This indicates that the applied ExB layer can manipulate a plasma layer in order to solve the communication blackout problem without disturbing the flow field.

Figure 10 shows the potential distribution of the $\mathrm{ExB}$ layer. As can be seen, the applied potential is mainly focused near the cathode. This means that the applied ExB layer can reduce plasma density only near the cathode.

\section{Discussion}

Figure 11 shows the plasma density distribution for OREX near the ExB layer as a function of applied magnetic-field strength and potential drop. As can be seen, the plasma density reduction increases with magnetic field strength and the highest plasma density reduction occurs when both the electric field and magnetic field are applied. This agrees with the previous study of an ExB layer as a mitigation method. ${ }^{3,6}$ Figure 11 also shows that the plasma density reduction improves significantly when the electric field is applied. This means that the ExB layer mitigation scheme gives better performance in the manipulation of the plasma layer than the magnetic window method which uses only a magnet.

Figure 11 reveals a plasma density reduction even when no voltage is applied to the cathode. The density reduction in this case occurs because of the magnetic field, which can generate a current density in the absence of an electric field. The generated current density is smaller than that in the electric field case, but it can give a Lorentz force for the plasma acceleration. When the electric field is applied, it gives a higher current density. In this case, the magnetic field helps to maintain a strong electric field. Therefore the electric field improves the plasma density reduction in the hypersonic plasma layer.

Figure 12 shows the maximum plasma density reduction in terms of applied magnetic-field strength and potential drop. The minimum required plasma density reduction is tabulated in Table 3 . At the $79.9 \mathrm{~km}$ reentry condition, OREX has a radio blackout for VHF, UHF, L- band, GPS frequency, and S-band. In order to solve radio blackout for the S-band, which is used for the data telemetry of OREX, the plasma density reduction should be stronger than 0.6. This density reduction can be obtained with a $0.3 \mathrm{~T}$ magnetic field in the ExB layer mitigation scheme. However, this configuration of the ExB layer still provides a weak plasma density reduction for the L-band and GPS frequency. Even a $0.5 \mathrm{~T}$ magnetic field can not solve a blackout for the L-band. However, the applied ExB layer with $0.5 \mathrm{~T}$ and $-1000 \mathrm{~V}$ potential drop gives a plasma density reduction of about 0.05 . In this case, the plasma density reduction satisfies the minimum required value for L-band and GPS communication. For the GPS frequency, signal attenuation becomes approximately $10 \mathrm{~dB}$ with the ExB layer mitigation scheme. This illustrates the possibility of an ExB layer mitigation scheme to solve radio blackout in the hypersonic flight condition.

The signal attenuations of OREX for several communication frequencies are shown in Fig. 13 under several magnetic and electric fields strength. The signal attenuation which is less than $10 \mathrm{~dB}$ gives a chance for communication through a plasma layer because the typical attenuation inside the signal reception module 
is approximately $10 \mathrm{~dB}$. As can be seen, a low-frequency radio waveband requires higher magnetic and electric field than a high-frequency radio waveband to solve a radio blackout.

However, the applied ExB layer increases total drag of the vehicle as shown in Table 4. As can be seen, the applied ExB layer decreases the aerodynamic drag but it introduces a Lorentz force drag, which is absent when the ExB layer is not applied. Therefore, the total drag of the vehicle is augmented when the ExB layer is applied. This phenomena shows another potential application of an ExB layer, which is known as an MHD parachute. ${ }^{31}$ The ExB layer can be useful for Mars entry where deceleration is difficult due to the thinner atmosphere, thus the classical parachute cannot efficiently decelerate the vehicle.

\section{Conclusion}

This study discussed the simulation of the ExB layer mitigation scheme in a hypersonic flow for the OREX reentry vehicle. Since OREX is representative of a typical blunt body reentry vehicle, the hypersonic flow simulation with the mitigation scheme is useful for illustrating the ExB layer mitigation scheme in a realistic operating condition.

The simulation results for OREX show that the applied ExB layer provides a reduced plasma density near the cathode in a hypersonic flow at a $79.9 \mathrm{~km}$ reentry condition. As expected, the plasma density reduction depends on the strength of the electric and magnetic fields. The maximum plasma density reduction occurs with a combination of the electric and magnetic fields. This fact indicates that to communicate through the plasma layer, the ExB layer mitigation scheme is a more effective solution for radio blackout than the magnetic window method which, though promising, is impractical. ${ }^{7}$

The configuration of the ExB layer can be determined in relation to the radio wave band for communication. As the flight data show, ${ }^{30}$ OREX experienced radio blackout at $80 \mathrm{~km}$ altitude. The simulation results at $79.9 \mathrm{~km}$ show that the plasma density of OREX is high enough to cause radio blackout for UHF, the L-band, and the S-band. For the S-band communication blackout, the ExB layer with a $0.3 \mathrm{~T}$ magnetic field provides a sufficient plasma density reduction to enable communication through the plasma layer. The ExB layer with $0.5 \mathrm{~T}$ and $-1000 \mathrm{~V}$ potential shows the possibility for communication through the plasma layer for the L band and GPS frequency. The applied ExB layer gives another effect beside manipulation of plasma density. The numerical result also shows that the total drag is increased when the ExB layer is applied, because the applied magnetic field introduces a Lorentz force drag. This gives another potential application of an ExB layer method as an MHD parachute for entry mission into planets with thin atmospheres, such as Mars.

\section{Acknowledgments}

This material is based upon work supported by the Department of Energy National Nuclear Security Administration under Award Number NA28614. This report was prepared as an account of work sponsored by an agency of the United States Government. Neither the United States Government nor any agency thereof, nor any of their employees, makes any warranty, express or implied, or assumes any legal liability or responsibility for the accuracy, completeness, or usefulness of any information, apparatus, product, or process disclosed, or represents that its use would not infringe privately owned rights. Reference herein to any specific commercial product, process, or service by trade name, trademark, manufacturer, or otherwise does not necessarily constitute or imply its endorsement, recommendation, or favoring by the United States Government or any agency thereof. The views and opinions of authors expressed herein do not necessarily state or reflect those of the United States Government or any agency thereof. The authors wish to thank Charles H. Jones, David P. Morris, Christopher N. Davis, Kristina M. Lemmer, Alec D. Gallimore, Brian E. Gilchrist, Peter Peterson, Tim Smith, Jonathan Zagel, and Kenneth G. Powell for very useful discussions on this subject.

\section{References}

\footnotetext{
${ }^{1}$ Scalabrin, L. C. and Boyd, I. D., "Numerical Simulation of Weakly Ionized Hypersonic Flow for Reentry Configurations," AIAA 2006-3773, June 2006, presented at the 9th AIAA/ASME Joint Thermophysics and Heat Transfer Conference, San Francisco, California.

${ }^{2}$ Rybak, J. P. and Churchill, R. J., "Progress in Re-entry Communications," IEEE Transactions on Aerospace and Electronic Systems, Vol. aes-7, No. 5, 1971, pp. 879-894.
} 
${ }^{3}$ Kim, M., Keidar, M., and Boyd, I. D., "Analysis of an Electromagnetic Mitigation Scheme for Reentry Telemetry Through Plasma," Journal of Spacecraft and Rockets, Vol. 45, No. 6, 2008, pp. 1223-1229.

${ }^{4}$ Starkey, R., Lewis, R., and Jones, C., "Electromagnetic Wave/Magnetoactive Plasma Sheath Interaction for Hypersonic Vehicle Telemetry Blackout Analysis," AIAA 2003-4167, June 2003, presented at 34th AIAA Plasmadynamics and Lasers Conference.

${ }^{5}$ Kim, M., Keidar, M., and Boyd, I. D., "Two-dimensional Model of an Electromagnetic Layer for the Mitigation of Communications Blackout," AIAA 2009-1232, Jan 2009, presented at the 47th AIAA Aerospace Sciences Meeting including The New Horizons Forum and Aerospace Exposition, Orlando, Florida.

${ }^{6}$ Keidar, M., Kim, M., and Boyd, I. D., "Electromagnetic Reduction of Plasma Density During Atmospheric Reentry and Hypersonic Flights," Journal of Spacecraft and Rockets, Vol. 45, No. 3, 2008, pp. 445-453.

${ }^{7}$ Hartunian, R. A., Stewart, G. E., Fergason, S. D., Curtiss, T. J., and Seibold, R. W., "Cause and Mitigations Radio Frequency (RF) Blackout During re-entry of Reusable Launch Vehicles," ATR 2007(5309)-1, AEROSPACE CORPORATION, 2007, ATR-2007(5309)-1.

${ }^{8}$ Fujino, T. and Ishikawa, M., "Numerical Simulation of Control of Plasma Flow With Magnetic Field for Thermal Protection in Earth Re-entry Flight," IEEE Transactions on Plasma Science, Vol. 34, No. 2, 2006, pp. 409-420.

${ }^{9}$ Scalabrin, L. C., Numerical Simulation of Weakly Ionized Hypersonic Flow Over Re-entry Capsules, 2007, PhD thesis, Department of Aerospace Engineering, University of Michigan.

${ }^{10}$ Yamamoto, Y. and Yoshioka, M., "CFD and FEM Coupling Analysis of OREX Aerothermodynamic Flight Data," AIAA 1995-2087, Jan 1995, presented at the 30th Thermophysics Conference, San Diego, CA.

${ }^{11}$ Shang, J. S., Menart, J., Kimmel, R. L., and Hayes, J., "Hypersonic Inlet with Plasma Induced Compression," AFRL$V A-W P-T P-2006-333$, Jan 2006

${ }^{12}$ Gaitonde, D. V., "Three-Dimensional Flow-Through Scramjet Simulation with MGD Energy-Bypass," AIAA Paper 2003-172, January 2003, presented at the 41st Aerospace Sciences Meeting, Reno, NV.

${ }^{13}$ Shutton, K. and Gnoffo, P. A., "Multi-component Diffusion with Application to Computational Aerothermodynamics," AIAA Paper 1998-2575, June 1998, presented at the 7th AIAA/ASME Joint Thermophysics and Heat Transfer Conference,Albuquerque, NM.

${ }^{14}$ White, F. M., Viscous Fluid Flow, McGraw-Hill Book Company, 3rd ed., 2006.

${ }^{15}$ Gnoffo, P. A., Gupta, R. N., and Shinn, J. L., "Conservation Equations and Physical Models for Hypersonic Air Flows in Thermal and Chemical Nonequilibrium," TP 2867, NASA, 1989.

${ }^{16}$ Dendy, R. O., Plasma Dynamics, Oxford University Press, 1990.

${ }^{17}$ Matsuo, T., Tadamatsu, A., and Shimasaki, M., "3-D Magnetohydrodynamic Field Computation of Supersonic Duct Flow of Weakly Ionized Plasma," Magnetic, IEEE Transactions on, Vol. 39, No. 3, May 2003, pp. 1444-1447.

${ }^{18}$ Meyer, R. X., "Magnetohydrodynamic-Hypersonic Flow in the Quasi-Newtonian Approximation," Reviews of Modern Physics, Vol. 32, No. 4, Oct 1960, pp. 1004-1007.

${ }^{19}$ Poggie, J., "Computational Studies of Magnetic Control in Hypersonic Flow," AIAA-2001-196, Jan 2001, presented at 39th Aerospace Sciences Meeting and Exhibit, Reno, NV.

${ }^{20}$ Dellar, P. J., "A Note on Magnetic Monopoles and the One-dimensional MHD Riemann Problem," Journal of Computational Physics, Vol. 172, No. 1, 2001, pp. 392-398.

${ }^{21} \mathrm{Kim}$, M., Boyd, I. D., and Keidar, M., "Modeling of Electromagnetic Manipulation of Plasma for Communication during Re-entry Flight," Journal of Spacecraft and Rockets, 2009, In progress.

${ }^{22}$ Lemmer, K. M., Gallimore, A. G., Smith, T. B., Davis, C. N., and Peterson, P., "Experimental Results from Application of an E x B Layer for Investigation of Communications Blackout Amelioration," Journal of Spacecraft and Rockets, 2009, In progress.

${ }^{23}$ Mitchner, M. and Kruger, C. H., Partially Ionized Gases, John Wiley, 1973.

${ }^{24}$ Fujino, T., Sugita, H., Mizuno, M., Funak, I., and Ishikawa, M., "Influences of Electrical Conductivity of Wall on Magnetohydrodynamic Control of Aerodynamic Heating," Journal of Spacecraft and Rockets, Vol. 43, No. 1, 2006, pp. 63-70.

${ }^{25}$ Fujino, T., Funak, I., Mizuno, M., Sugita, H., and Ishikawa, M., "Developing Field Emitter Array Cathode Systems for Electrodynamic Tether Propulsion," AIAA Paper 2004-2561, June 2004, presented at the 35th AIAA Plasmadynamics and Lasers Conference, Portland, Oregon.

${ }^{26}$ Borghi, C. A., Carraro, M. R., and Cristofolini, A., "Numerical Modeling of MHD Interaction in the Boundary Layer of Hypersonic Flows," IEEE Transactions on Magnetics, Vol. 39, No. 3, 2003, pp. 1507-1510.

${ }^{27}$ JAXA(Japan Aerospace Exploration Agency) hompage. http://www.jaxa.jp/.

${ }^{28}$ Leung, E., Hilal, M., Parmer, J., and Peck, S., "Lightweight Magnet for Space Applications," Magnetics, IEEE Transactions on, Vol. 23, No. 2, March 1987, pp. 1331-1335.

${ }^{29}$ Yamamoto, Y. and Yoshioka, M., "CFD and FEM Coupling Analysis of OREX Aerothermodynamic Flight Data," AIAA 95-2087, June 1995, presented at 30th Thermophysics Conference, San Diego, CA.

${ }^{30}$ Inouye, Y., "OREX Flight - Quick Report and Lessons Learned," Aerothermodynamics for space vehicles, edited by J. J. Hunt, Vol. 367 of ESA Special Publication, 1995, pp. 271-278.

${ }^{31}$ Bityurin, V. A., Bocharov, A. N., and Popov, N. A., "Non-Equilibrium Effects in MHD Parachute Concept Induced Electric Field Effects," AIAA 2009-1230, Jan 2009, presented at the 47th AIAA Aerospace Sciences Meeting including The New Horizons Forum and Aerospace Exposition, Orlando, Florida. 
Table 1. Relative mass of electrons by comparison with atoms and molecules

\begin{tabular}{|c|c|}
\hline Atoms $/$ Molecules & Relative mass of electron, $\frac{m_{e}}{m_{i}}$ \\
\hline $\mathrm{O}$ & $3.4290 \times 10^{-5}$ \\
$\mathrm{O}_{2}$ & $1.7145 \times 10^{-5}$ \\
$\mathrm{~N}$ & $3.9168 \times 10^{-5}$ \\
$\mathrm{~N}_{2}$ & $1.9584 \times 10^{-5}$ \\
$\mathrm{NO}$ & $1.8284 \times 10^{-5}$ \\
\hline
\end{tabular}

Table 2. Freestream conditions and wall temperature of OREX for several altitude conditions ${ }^{29}$

\begin{tabular}{|c|c|c|c|c|}
\hline Altitude & Velocity & Temperature & Pressure & Wall Temperature \\
$\mathrm{H}(\mathrm{km})$ & $U_{\infty}(\mathrm{m} / \mathrm{s})$ & $T_{\infty}(\mathrm{K})$ & $P_{\infty}(\mathrm{Pa})$ & $T_{w}(\mathrm{~K})$ \\
\hline 92.82 & 7454.1 & 188.70 & 0.163 & 492 \\
84.01 & 7415.9 & 188.90 & 0.594 & 587 \\
79.90 & 7360.2 & 198.64 & 1.052 & 690 \\
71.73 & 7049.2 & 214.98 & 4.023 & 1078 \\
67.66 & 6720.3 & 225.99 & 7.892 & 1251 \\
63.60 & 6223.4 & 237.14 & 14.02 & 1413 \\
59.60 & 5561.6 & 248.12 & 23.60 & 1519 \\
55.74 & 4759.1 & 258.74 & 39.48 & 1571 \\
51.99 & 3873.4 & 268.20 & 63.48 & 1557 \\
48.40 & 3000.0 & 270.65 & 98.50 & 1501 \\
\hline
\end{tabular}

Table 3. The required plasma density reduction for OREX at $79.9 \mathrm{~km}$ reentry condition

\begin{tabular}{|c|c|c|c|c|c|c|}
\hline $\begin{array}{c}\text { VHF } \\
300 \mathrm{MHz}\end{array}$ & $\begin{array}{c}\text { UHF } \\
3000 \mathrm{MHz}\end{array}$ & $\begin{array}{c}\text { L band } \\
2 \mathrm{GHz}\end{array}$ & $\begin{array}{c}\text { GPS } \\
1.4 \mathrm{GHz}\end{array}$ & $\begin{array}{c}\text { S band } \\
4 \mathrm{GHz}\end{array}$ & $\begin{array}{c}\mathrm{X} \text { band } \\
12 \mathrm{GHz}\end{array}$ & $\begin{array}{c}\mathrm{K}_{u} \text { band } \\
18 \mathrm{GHz}\end{array}$ \\
\hline 0.004 & 0.3497 & 0.1486 & 0.0728 & 0.5945 & No blackout & No blackout \\
\hline
\end{tabular}

Table 4. The total drag force of OREX

\begin{tabular}{|ccc||ccc|}
\hline \multicolumn{3}{|c|}{ Without ExB layer } & \multicolumn{2}{c|}{ With ExB layer $(\mathrm{B}=0.1 \mathrm{~T}$ and $\Delta \phi=-800 \mathrm{~V})$} \\
Aerodynamic drag & Lorenz force drag & Total drag & Aerodynamic drag & Lorentz force drag & Total drag \\
\hline $420 \mathrm{~N}$ & $0 \mathrm{~N}$ & $420 \mathrm{~N}$ & $332 \mathrm{~N}$ & $204 \mathrm{~N}$ \\
\hline
\end{tabular}




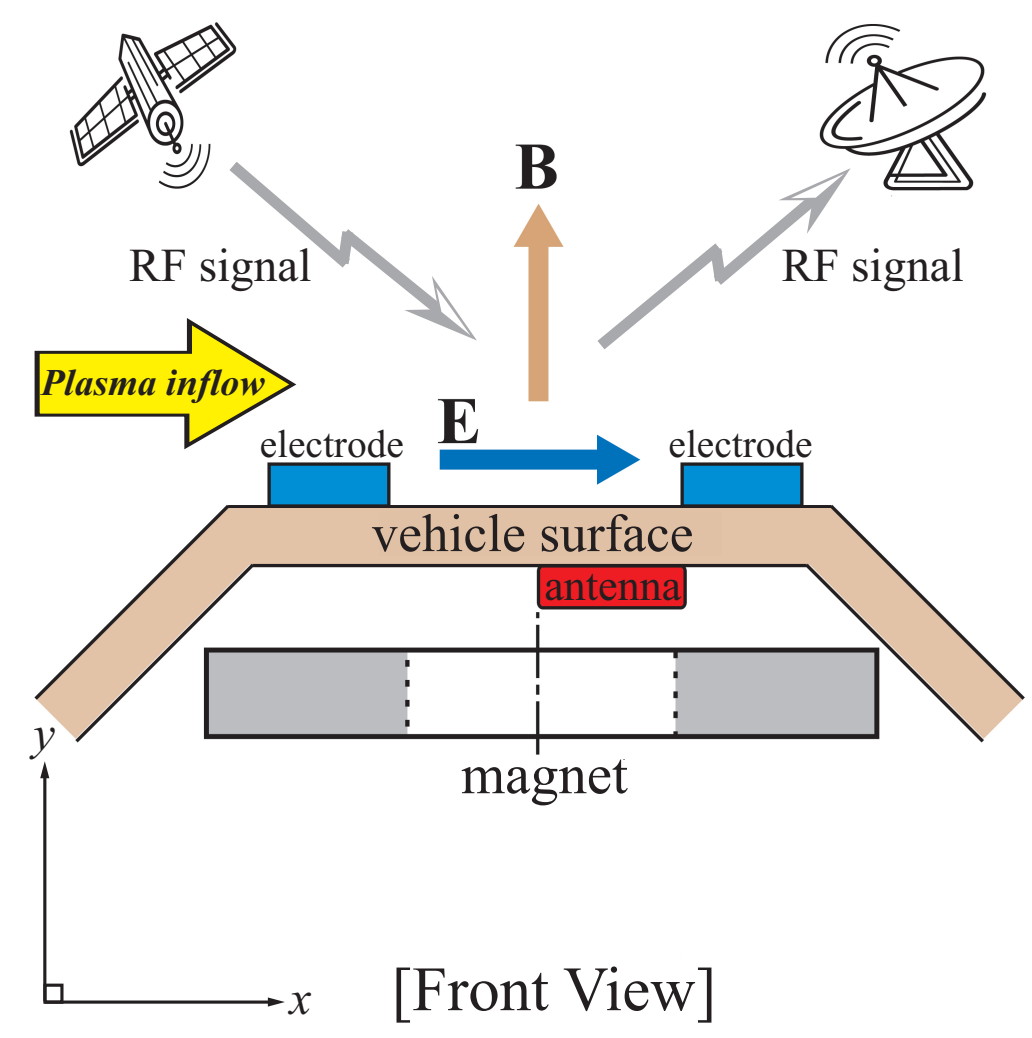

Figure 1. Schematic of an applied ExB field for manipulating plasma in a hypersonic boundary layer

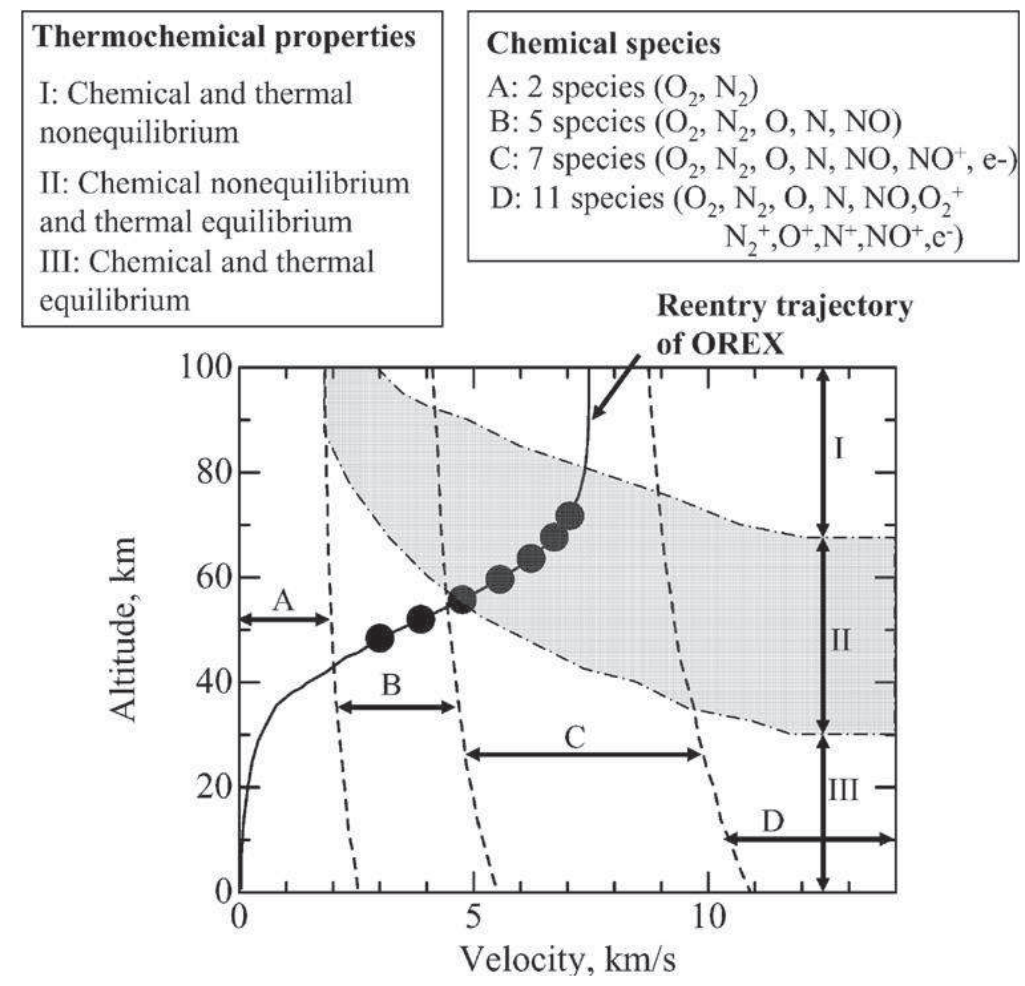

Figure 2. Thermochemical properties of gas in the shock layer in reentry flight for OREX [taken from $\left.{ }^{8}\right]$ 


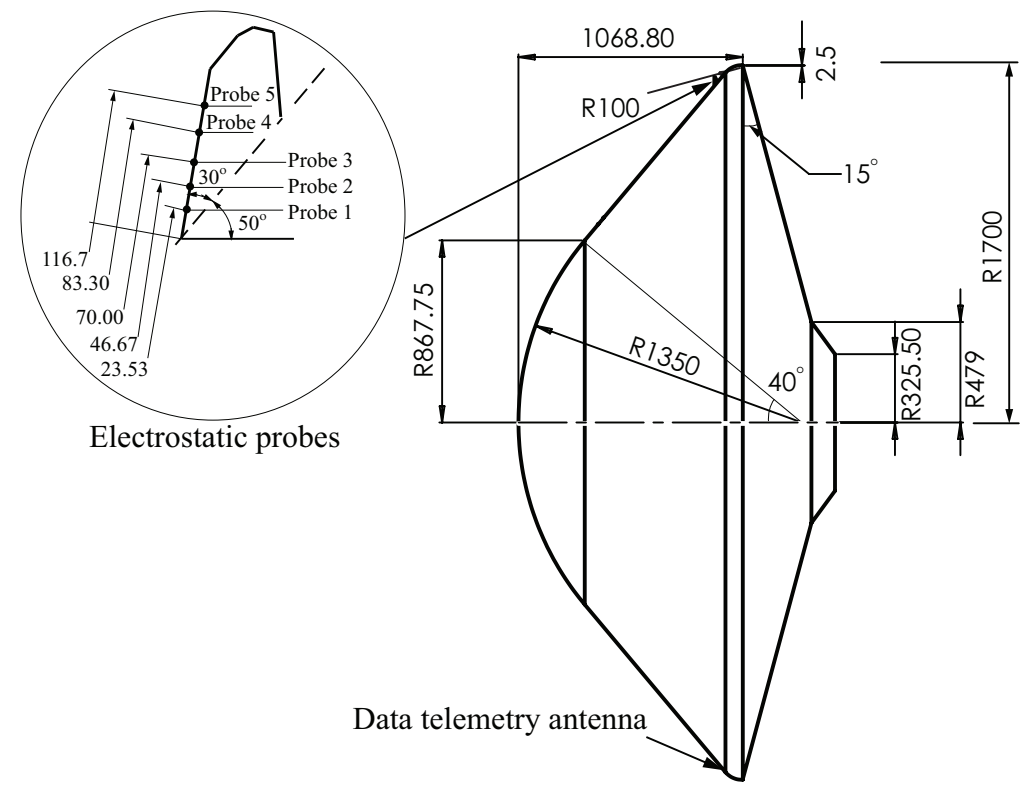

Figure 3. Geometry of OREX where the units are mm

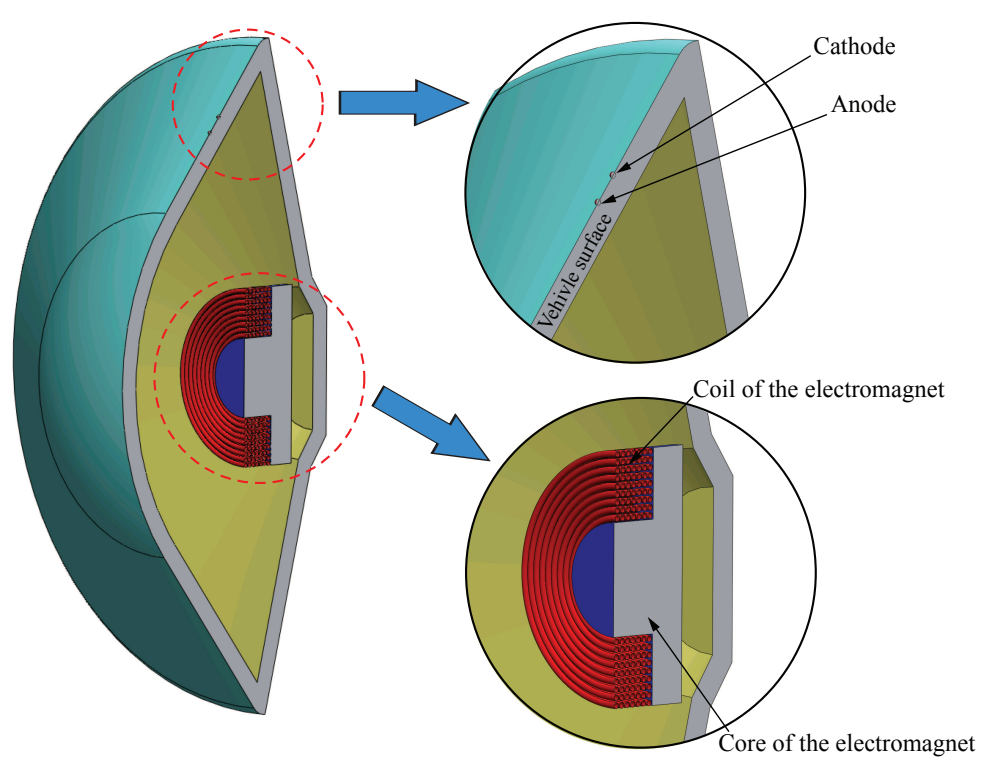

Figure 4. Configuration of OREX with the electrode and electromagnet which provide the ExB layer as the blackout mitigation scheme. 


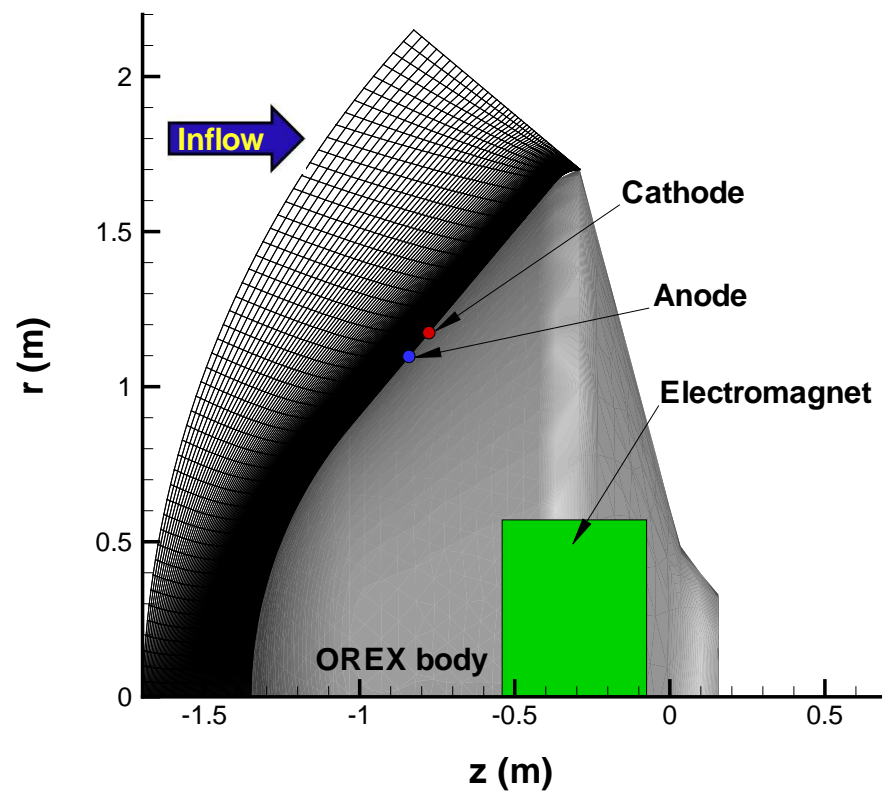

Figure 5. Mesh for the two-dimensional OREX simulation with the ExB layer with a schematic of the ExB layer configuration 


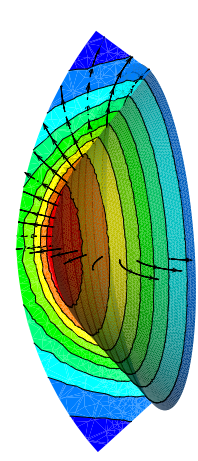

(a)

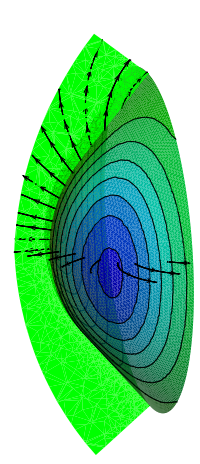

(c)

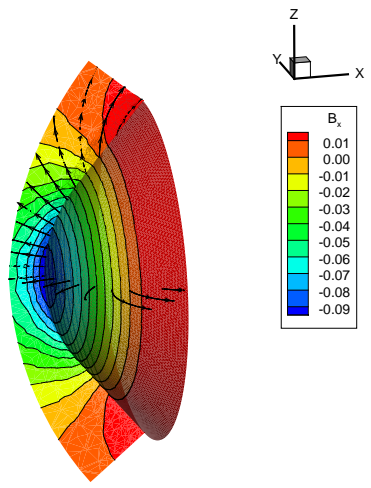

(b)

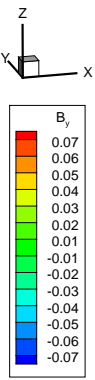

(d)

Figure 6. Configuration of the applied magnetic field: (a) Magnetic field strength, $B$, in $\mathbf{T}$ with the magnetic field lines. (b) $\mathrm{x}$-direction magnetic field, $B_{x}$, in $\mathbf{T}$ with the magnetic field lines. (c) y-direction magnetic field, $B_{y}$, in $\mathbf{T}$ with the magnetic field lines. (d) z-direction magnetic field, $B_{z}$, in $\mathbf{T}$ with the magnetic field lines. 


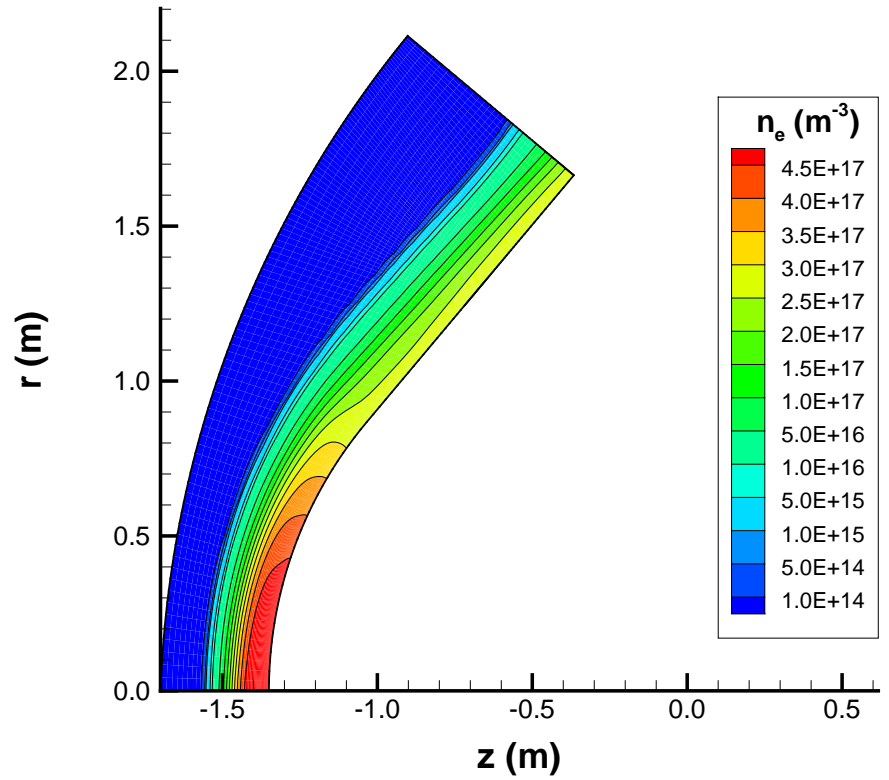

Figure 7. Distribution of the electron number density without the ExB layer mitigation scheme.

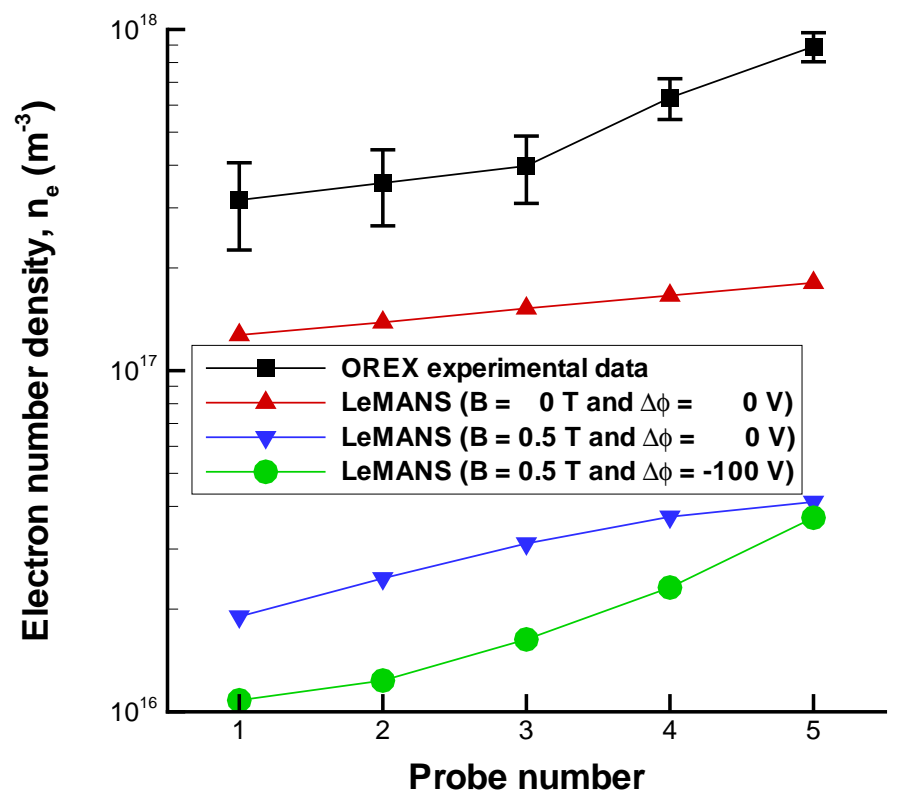

Figure 8. The comparison of the OREX measurement and the simulation result of LeMANS for electron number density. 


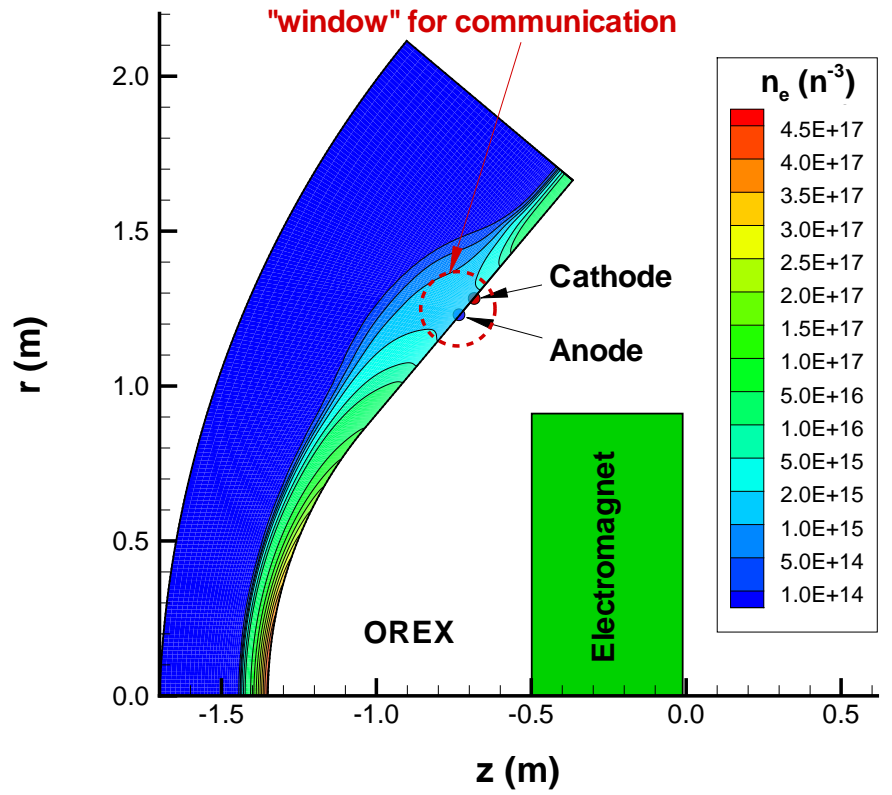

Figure 9. Distribution of the electron number density with the ExB layer mitigation scheme. The applied magnetic field is $0.1 \mathrm{~T}$ with $-800 \mathrm{~V}$ a potential drop.

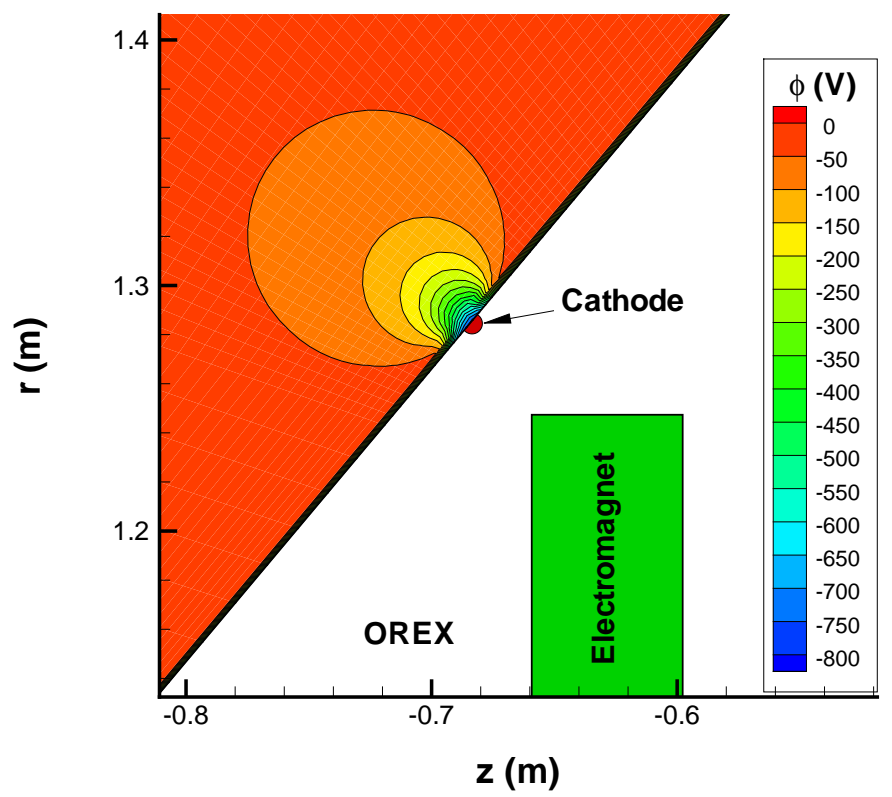

Figure 10. Distribution of potential: the anode is grounded at $0 \mathrm{~V}$ and the cathode is at $-800 \mathrm{~V}$ 


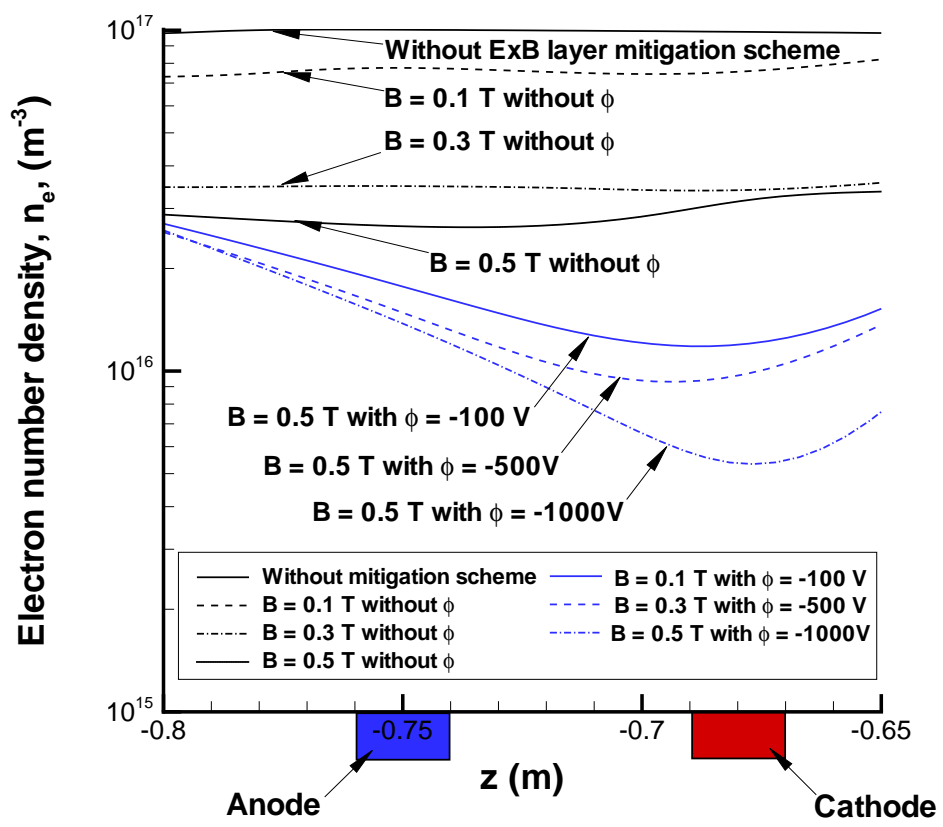

Figure 11. The electron number density distribution near the surface of OREX for several operational conditions of an ExB mitigation scheme.

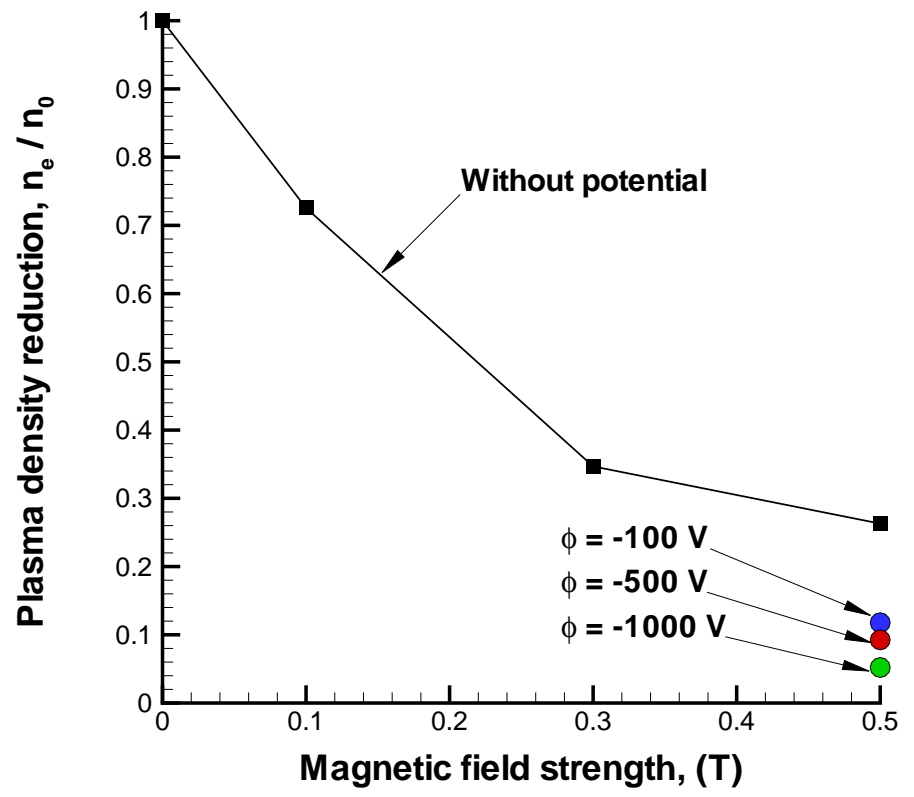

Figure 12. The maximum plasma density reduction by an ExB layer in a hypersonic flight condition 


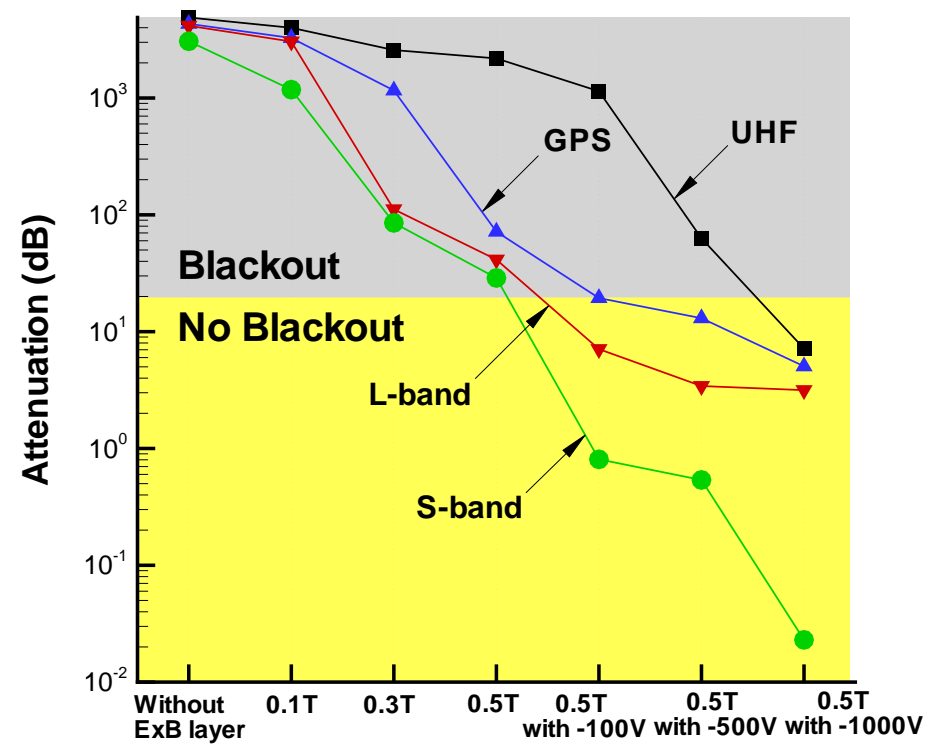

Figure 13. The signal attenuation of OREX in terms of the ExB layer configuration for several communication frequencies. 